\title{
A RELATION BETWEEN NÖRLUND AND ABEL SUMMABILITIES
}

\author{
MEHMET A. SARIGÖL
}

(Communicated by Andrew Bruckner)

\begin{abstract}
This paper gives a necessary and sufficient condition in order that every series summable by a Nörlund method with $p_{n}=o\left(P_{n}\right)$ as $n \rightarrow \infty$ should be summable by Abel's method to the same sum and so extends two theorems of Zygmund.
\end{abstract}

\section{INTRODUCTION}

Let $z$ designate a complex variable; then the series $\sum a_{n}$, with partial sums $s_{n}$, is summable to $s$ by Abel's method if

$$
\lim _{z \rightarrow 1} \sum_{n=0}^{\infty} a_{n} z^{n}=\lim _{z \rightarrow 1}(1-z) \sum_{n=0}^{\infty} s_{n} z^{n}=s
$$

as $z \rightarrow 1$ along any path lying between two chords of the unit circle which pass through $z=1$ [3].

Let $\left(p_{n}\right)$ denote a sequence of complex numbers such that $P_{n}=p_{0}+p_{1}+$ $\cdots+p_{n} \neq 0$ for all $n \geq 0$. The series $\sum a_{n}$ is Nörlund summable $\left(N, p_{n}\right)$ to $s$ if the sequence-to-sequence

$$
t_{n}=\sum_{v=0}^{n} p_{n-v} P_{n}^{-1} s_{v}
$$

$\rightarrow s$ as $n \rightarrow \infty$. The case $p_{n}=A_{n}^{\alpha-1}$, where $A_{n}^{\alpha-1}$ is defined from the identity

$$
\sum_{n=0}^{\infty} A_{n}^{\alpha-1} x^{n}=(1-x)^{-\alpha}
$$

is Cesàro summability $(C, \alpha)$ (see $[1,4])$. The inverse transformation to $(2)$ is given by

$$
s_{n}=\sum_{v=0}^{n} c_{n-v} P_{v} t_{v}
$$

Received by the editors December 28, 1992.

1991 Mathematics Subject Classification. Primary 40G05, 40G10; Secondary 40D25.

This paper was supported by TBAG-CG2 (TÜBITAK). 
where $\left(c_{n}\right)$ is defined by the formal power series relation

$$
\sum_{n=0}^{\infty} c_{n} x^{n} \cdot \sum_{v=0}^{\infty} p_{v} x^{v}=c(x) \cdot p(x)=1
$$

We write

$$
P(z)=\sum_{n=0}^{\infty} P_{n} z^{n} \quad \text { and } \quad P^{*}(z)=\sum_{n=0}^{\infty}\left|P_{n}\right|\left|z^{n}\right|
$$

We now prove the following theorem.

Theorem A. Let $\left(N, p_{n}\right)$ be a Nörlund method (not necessarily regular) such that

$$
p_{n}=o\left(P_{n}\right) \text { as } n \rightarrow \infty .
$$

Then so that every series Nörlund summable $\left(N, p_{n}\right)$ is Abel summable to the same sum it is necessary and sufficient that

(i) $|P(z)| \rightarrow \infty$ as $z \rightarrow 1$, and

(ii) $\quad P^{*}(z)=O(|P(z)|)$ as $z \rightarrow 1$,

where $z$ approaches $z=1$ in the angular region described.

Proof of the theorem. Suppose $t_{n} \rightarrow s$ as $n \rightarrow \infty$, where $t_{n}$ is the $\left(N, p_{n}\right)$ mean of $\sum a_{n}$. It follows from (6) that the series in (5) and

$$
c(z)=\sum_{n=0}^{\infty} c_{n} z^{n}
$$

are convergent for all $|z|<1$, where the $c_{n}$ 's are related by (4). Also because of convergence of $\left(t_{n}\right), \sum_{n=0}^{\infty} P_{n} t_{n} z^{n}$ are absolutely convergent for $|z|<1$. Now let $\left(z_{n}\right)$ be any sequence of points within the unit circle and the angle, tending to 1 . Then by (3) and (4),

$$
\begin{aligned}
f\left(z_{n}\right) & =\left(1-z_{n}\right) \sum_{v=0}^{\infty} s_{v} z_{v}^{n}=\left(1-z_{n}\right) \sum_{v=0}^{\infty}\left(\sum_{i=0}^{v} c_{v-i} P_{i} t_{i}\right) z_{n}^{v} \\
& =\left(1-z_{n}\right) \sum_{v=0}^{\infty} P_{v} t_{v} z_{n}^{v} \cdot \sum_{i=0}^{\infty} c_{i} z_{n}^{i}=\left(1-z_{n}\right)\left(p\left(z_{n}\right)\right)^{-1} \sum_{v=0}^{\infty} P_{v} t_{v} z_{n}^{v} .
\end{aligned}
$$

On the other hand, since $(1-z) P(z)=p(z)$ for $|z|<1$, we have

$$
f\left(z_{n}\right)=\left(P\left(z_{n}\right)\right)^{-1} \sum_{v=0}^{\infty} P_{v} t_{v} z_{n}^{v}, \quad|z|<1 ;
$$

i.e., $\left(f\left(z_{n}\right)\right)$ is $T=\left(a_{n v}\right)$-transform sequence of $\left(t_{n}\right)$, where

$$
a_{n v}=\frac{P_{v} z_{n}^{v}}{P\left(z_{n}\right)}
$$


for all $n, v$. Hence, it suffices to show that $f\left(z_{n}\right) \rightarrow s$ as $n \rightarrow \infty$ if and only if the matrix $T$ is regular. Under the sufficiency hypothesis of the theorem, $\lim _{n \rightarrow \infty} a_{n v}=0$ for each $v$, since

$$
\lim _{n \rightarrow \infty} a_{n v}=P_{v} \lim _{n \rightarrow \infty}\left(P\left(z_{n}\right)\right)^{-1}=0,
$$

by (7i). Moreover, we have $\sum_{v=0}^{\infty} a_{n v}=\left(P\left(z_{n}\right)\right)^{-1} \sum_{v=0}^{\infty} P_{v} z_{n}^{v}=1$ and finally

$$
\sum_{v=0}^{\infty}\left|a_{n v}\right|=\left(\left|P\left(z_{n}\right)\right|\right)^{-1} \sum_{v=0}^{\infty}\left|P_{v}\right|\left|z_{n}^{v}\right|=P^{*}\left(z_{n}\right)\left(\left|P\left(z_{n}\right)\right|\right)^{-1}=O(1)
$$

as $n \rightarrow \infty$, by (7ii). Hence $T=\left(a_{n v}\right)$ is a regular matrix; it is also easy to see from the above that the conditions of the theorem are necessary.

\section{Applications}

In particular, if $p_{n}=A_{n}^{\alpha-1}, \alpha>-1$, for all $n$, then $P(z)=(1-z)^{-\alpha-1}$ and $P^{*}(z)=(1-|z|)^{-\alpha-1}$. It is known [2] that there exists a constant $M$ such that

$$
P^{*}(z) \cdot(|P(z)|)^{-1}=\left(|1-z|(1-|z|)^{-1}\right)^{\alpha+1} \leq M
$$

for all $z$ in the neighbourhood of the point 1 . Therefore, Zygmund's results (Theorems 1.33 and 1.34 of [5]) follow from Theorem A.

On the other hand, as a corollary we have Theorem 18 of Hardy [1, p. 65] in the following form.

Theorem B. Let $\left(N, p_{n}\right)$ be a Nörlund method such that $p_{0}>0, p_{n} \geq 0$, and $p_{n}=o\left(P_{n}\right)$ as $n \rightarrow \infty$. If a series is summable $\left(N, p_{n}\right)$ to sum $s$, then (1) holds as $x$ tends to 1 along the real axis.

Proof. Since $\sum_{n=0}^{\infty} P_{n}$ is divergent, it follows from Abel's theorem on power series that $P(x) \rightarrow \infty$ as $x \rightarrow 1-0$. Moreover, $P^{*}(x)=P(x)$ in the neighbourhood of point 1 . Thus the theorem is obtained by Theorem A.

\section{REFERENCES}

1. G. H. Hardy, Divergent series, Oxford Univ. Press, Oxford, 1949.

2. K. Knopp, Theory and application of infinite series, Blackie and Son, London and Glasgow, 1944.

3. G. M. Petersen, Regular matrix transformation, McGraw-Hill, England, 1966.

4. A. Peyerimhoff, Lectures on summability, Lecture Notes in Math., vol. 107, Springr-Verlag, Berlin, Heidelberg, and New York, 1969.

5. A. Zygmund, Trigonometric series, Cambridge Univ. Press, Cambridge, 1959.

Department of Mathematics, University of Erciyes, 38039 Kayseri, Turkey 